\title{
Multi-Interval Discretization of Continuous Attributes for Label Ranking
}

\author{
Cláudio Rebelo de Sá ${ }^{1,2}$, Carlos Soares ${ }^{1}$, and Arno Knobbe ${ }^{2}$, Paulo Azevedo ${ }^{3}$, \\ Alípio Mário Jorge ${ }^{4}$ \\ 1 INESCTEC, Porto, Portugal \\ 2 LIACS, Universiteit Leiden, Netherlands \\ 3 CCTC, Departamento de Informática, Universidade do Minho \\ 4 DM - Faculdade de Ciencias, Universidade do Porto \\ claudio@liacs.nl, csoares@fe.up.pt, knobbe@liacs.nl, pja@uminho.pt, \\ amjorge@fc.up.pt
}

\begin{abstract}
Label Ranking (LR) problems, such as predicting rankings of financial analysts, are becoming increasingly important in data mining. While there has been a significant amount of work on the development of learning algorithms for LR in recent years, pre-processing methods for LR are still very scarce. However, some methods, like Naive Bayes for LR and APRIORI-LR, cannot deal with real-valued data directly. As a make-shift solution, one could consider conventional discretization methods used in classification, by simply treating each unique ranking as a separate class. In this paper, we show that such an approach has several disadvantages. As an alternative, we propose an adaptation of an existing method, MDLP, specifically for LR problems. We illustrate the advantages of the new method using synthetic data. Additionally, we present results obtained on several benchmark datasets. The results clearly indicate that the discretization is performing as expected and in some cases improves the results of the learning algorithms.
\end{abstract}

\section{Introduction}

A reasonable number of learning algorithms has been created or adapted for LR in recent years $[15,11,7,4,5]$. LR studies the problem of learning a mapping from instances to rankings over a finite number of predefined labels. It can be considered as a variant of the conventional classification problem [3]. However, in contrast to a classification setting, where the objective is to assign examples to a specific class, in LR we are interested in assigning a complete preference order of the labels to every example. An additional difference is that the true (possibly partial) ranking of the labels is available for the training examples.

Discretization, from a general point of view, is the process of partitioning a given interval into a set of discrete sub-intervals. It is usually used to split continuous intervals into two or more sub-intervals which can be treated as nominal values. This pre-processing technique enables the application to numerical data of learning methods that are otherwise unable to process them directly (like 
Bayesian Networks and Rule Learning methods). In theory, a good discretization should have a good balance between the loss of information and the number of partitions [13].

Discretization methods come in two flavors, depending on whether they do, or do not involve target information. These are usually referred to as supervised and unsupervised, respectively. Previous research found that the supervised methods produce more accurate discretization than unsupervised methods [8]. To the best of our knowledge, there are no supervised discretization methods for LR. Hence, our proposal for ranking-sensitive discretization is a useful contribution for the LR community.

We propose an adaptation of a well-known supervised discretization method, the Minimum Description Length Principle (MDLP) [10], for LR. The method uses an entropy-like measure for a set of rankings based on a similarity measure for rankings. Despite this basic heuristic approach, the results observed show that the method is behaves as expected in an LR setting.

The paper is organized as follows: Section 2 introduces the LR problem and the task of association rule mining. Section 3 introduces discretization and Section 4 describes the method proposed here. Section 5 presents the experimental setup and discusses the results. Finally, Section 6 concludes this paper.

\section{Label Ranking}

The LR task is similar to classification. In classification, given an instance $x$ from the instance space $\mathbb{X}$, the goal is to predict the label (or class) $\lambda$ to which $x$ belongs, from a pre-defined set $\mathcal{L}=\left\{\lambda_{1}, \ldots, \lambda_{n}\right\}$. In LR the goal is to predict the ranking of the labels in $\mathcal{L}$ that are associated with $x$. We assume that the ranking is a total order over $\mathcal{L}$ defined on the permutation space $\Omega$. A total order can be seen as a permutation $\pi$ of the set $\{1, \ldots, n\}$, such that $\pi(a)$ is the position of $\lambda_{a}$ in $\pi .^{5}$

As in classification, we do not assume the existence of a deterministic $\mathbb{X} \rightarrow \Omega$ mapping. Instead, every instance is associated with a probability distribution over $\Omega$. This means that, for each $x \in \mathbb{X}$, there exists a probability distribution $P(\cdot \mid x)$ such that, for every $\pi \in \Omega, P(\pi \mid x)$ is the probability that $\pi$ is the ranking associated with $x$. The goal in LR is to learn the mapping $\mathbb{X} \rightarrow \Omega$. The training data is a set of instances $T=\left\{\left\langle x_{i}, \pi_{i}\right\rangle\right\}, i=1, \ldots, n$, where $x_{i}$ are the independent variables describing instance $i$ and $\pi_{i}$ is the corresponding target ranking.

Given an instance $x$ with label ranking $\pi$, and the ranking $\hat{\pi}$ predicted by an LR model, we need to evaluate the accuracy of the prediction. For that, we need a loss function on $\Omega$. One such function is the number of discordant label pairs,

$$
D(\pi, \hat{\pi})=\#\{(i, j) \mid \pi(i)>\pi(j) \wedge \hat{\pi}(i)<\hat{\pi}(j)\}
$$

which, if normalized to the interval $[-1,1]$, is equivalent to Kendall's $\tau$ coefficient [12], which is a correlation measure where $D(\pi, \pi)=1$ and $D\left(\pi, \pi^{-1}\right)=-1$ (here, $\pi^{-1}$ denotes the inverse order of $\pi$ ).

\footnotetext{
${ }_{5}$ This assumption may be relaxed [3].
} 
The accuracy of a model can be estimated by averaging this function over a set of examples. This measure has been used for evaluation in recent LR studies [3] and, thus, we will use it here as well. However, other correlation measures, like Spearman's rank correlation coefficient [16], can be used equally well, were one so inclined.

Given the similarities between LR and classification, one could consider workarounds that treat the label ranking problem essentially as a classification problem.

Let us define a basic pre-processing method, which replaces the rankings with classes, as Ranking As Class (RAC).

$$
\forall \pi_{i} \in \Omega, \pi \rightarrow \lambda_{i}
$$

This method has a number of disadvantages, as discussed in the next section, but it allows the use of all pre-processing and prediction methods for classification in LR problems. However, as we show in this work, this approach is neither the most effective nor the most accurate.

\subsection{Association Rules for Label Ranking}

Label Ranking Association Rules (LRAR) [7] are a straightforward adaptation of class Association Rules (CAR):

$$
A \rightarrow \pi
$$

where $A \subseteq \operatorname{desc}(\mathbb{X})$ and $\pi \in \Omega$. Similar to how predictions are made in CBA (Classification Based on Associations) [14], when an example matches the rule $A \rightarrow \pi$, the predicted ranking is $\pi$.

If the RAC method is used, the number of classes can be extremely large, up to a maximum of $k$, where $k$ is the size of the set of labels, $\mathcal{L}$. This means that the amount of data required to learn a reasonable mapping $\mathbb{X} \rightarrow \Omega$ is too big.

Secondly, this approach does not take into account the differences in nature between label rankings and classes. In classification, two examples either have the same class or not, whereas in LR some rankings are more similar than others, as they only differ in one or two swaps or labels. In this regard, LR is more similar to regression than to classification. This property can be used in the induction of prediction models. In regression, a large number of observations with a given target value, say 5.3, increases the probability of observing similar values, say 5.4 or 5.2 , but not so much for very different values, say -3.1 or 100.2 . A similar reasoning was done for LR in [7]. Let us consider the case of a data set in which ranking $\pi_{a}=\{A, B, C, D, E\}$ occurs in $1 \%$ of the examples. Treating rankings as classes would mean that $P\left(\pi_{a}\right)=0.01$. Let us further consider that the rankings $\pi_{b}=\{A, B, C, E, D\}, \pi_{c}=\{B, A, C, D, E\}$ and $\pi_{d}=\{A, C, B, D, E\}$ occur in $50 \%$ of the examples. Taking into account the stochastic nature of these rankings [3], $P\left(\pi_{a}\right)=0.01$ seems to underestimate the probability of observing $\pi_{a}$. In other words it is expected that the observation of $\pi_{b}, \pi_{c}$ and $\pi_{d}$ increases 
the probability of observing $\pi_{a}$ and vice-versa, because they are similar to each other.

This affects even rankings which are not observed in the available data. For example, even though $\pi_{e}=\{A, B, D, C, E\}$ is not present in the data set it would not be entirely unexpected to see it in future data.

Similarity-based Support and Confidence Given a measure of similarity between rankings $s\left(\pi_{a}, \pi_{b}\right)$, the support of the rule $A \rightarrow \pi$ is defined as follows:

$$
\sup _{l r}(A \rightarrow \pi)=\frac{\sum_{i: A \subseteq \operatorname{desc}\left(x_{i}\right)} s\left(\pi_{i}, \pi\right)}{n}
$$

This is, essentially, assigning a weight to each target ranking in the training, $\pi_{i}$, data that represents its contribution to the probability that $\pi$ may be observed. Some instances $x_{i} \in \mathbb{X}$ give full contribution to the support count (i.e., 1 ), while others may give partial or even a null contribution.

Any function that measures the similarity between two rankings or permutations can be used, such as Kendall's $\tau$ or Spearman's $\rho$. The function used here is of the form:

$$
s\left(\pi_{a}, \pi_{b}\right)=\left\{\begin{array}{rr}
s^{\prime}\left(\pi_{a}, \pi_{b}\right) & \text { if } s^{\prime}\left(\pi_{a}, \pi_{b}\right) \geq \theta_{\text {sup }} \\
0 & \text { otherwise }
\end{array}\right.
$$

where $s^{\prime}$ is a similarity function. This general form assumes that below a given threshold, $\theta_{\text {sup }}$, it is not useful to discriminate between different similarity values, as they are so different from $\pi_{a}$. This means that, the support $\sup$ of $\left\langle A, \pi_{a}\right\rangle$ will have contributions from all the ruleitems of the form $\left\langle A, \pi_{b}\right\rangle$, for all $\pi_{b}$ where $s^{\prime}\left(\pi_{a}, \pi_{b}\right)>\theta_{\text {sup }}$.

The confidence of a rule $A \rightarrow \pi$ is obtained simply by replacing the measure of support with the new one.

$$
\operatorname{conf}_{l r}(A \rightarrow \pi)=\frac{\sup _{l r}(A \rightarrow \pi)}{\sup (A)}
$$

Given that the loss function that we aim to minimize is known beforehand, it makes sense to use it to measure the similarity between rankings. Therefore, we use Kendall's $\tau$. In this case, we think that $\theta_{\text {sup }}=0$ would be a reasonable value, given that it separates the negative from the positive contributions. Table 1 shows an example of a label ranking dataset represented following this approach.

To present a more clear interpretation, the example given in Table 1 , the instance

$$
(\{A 1=L, A 2=X L, A 3=S\})(T I D=1)
$$

contributes 1 to the support count of the ruleitem:

$$
\left\langle\{A 1=L, A 2=X L, A 3=S\}, \pi_{3}\right\rangle
$$


Table 1. An example of a label ranking dataset to be processed by the APRIORI-LR algorithm.

\begin{tabular}{lrlrrrr} 
& & & & $\pi_{1}$ & $\pi_{2}$ & $\pi_{3}$ \\
TID & A1 & A2 & A3 & $(1,3,2)$ & $(2,1,3)$ & $(2,3,1)$ \\
\hline $\mathbf{1}$ & L & XL & S & 0.33 & 0.00 & 1.00 \\
$\mathbf{2}$ & XXL & XS & S & 0.00 & 1.00 & 0.00 \\
$\mathbf{3}$ & L & XL XS & 1.00 & 0.00 & 0.33
\end{tabular}

The same instance will also give a small contribution of 0.33 to the support count of the ruleitem

$$
\left\langle\{A 1=L, A 2=X L, A 3=S\}, \pi_{1}\right\rangle
$$

given their similarity. On the other hand, no contribution is given to the count used for the support of ruleitem

$$
\left\langle\{A 1=L, A 2=X L, A 3=S\}, \pi_{2}\right\rangle
$$

which makes sense as they are clearly different.

\section{Discretization}

Several Data Mining (DM) algorithms can improve their performance by using discretized versions of continuous-valued attributes [9]. Given that a large number of algorithms, like the Naive Bayes classifier, cannot work without discretized data [13] and the majority of real datasets have continuous variables, a good discretization method can be very relevant for the accuracy of the models. Discretization methods deal with continuous variables by partitioning them into intervals or ranges. Then, each of these intervals can be interpreted as a nominal value by $\mathrm{DM}$ algorithms.

The main issue in discretization is the choice of the intervals because a continuous variable can be discretized in an infinite number of ways. An ideal discretization method finds a reasonable number ${ }^{6}$ of cut points that split the data into meaningful intervals. For classification datasets, a meaningful interval should be coherent with the class distribution along the variable.

Discretization approaches can be divided into two groups:

Supervised vs Unsupervised When dealing with classification datasets the discretization methods can use the values of the target variable or not. These are referred to as supervised and unsupervised respectively. The unsupervised methods ignore the classes of the objects and divide the interval into a user-defined number of bins. Supervised methods take into account the distribution of the class labels in the discretization process. Previous research states that the supervised methods tend to produce better discretizations than unsupervised methods [8].

${ }^{6}$ An extreme discretization approach would create one nominal value for each continuous value but this is naturally not a reasonable approach. 
Top-down vs Bottom-up Discretization methods with a Top-down or Bottom-up approach start by sorting the dataset with respect to the variable which will be discretized. In the Top-down approach, the method starts with an interval containing all points. Then, it recursively splits the intervals into sub-intervals, until a stopping criteria is reached.

In the Bottom-up approach, the method starts with the maximum number of intervals (i.e., one for each value) and then iteratively merges them recursively until a stopping criteria is satisfied.

\subsection{Entropy based methods}

Several methods, such as $[6,10]$, perform discretization by optimizing entropy. In classification, class entropy is a measure of uncertainty in a finite interval of classes and it can be used in the search of candidate partitions. A good partition is such that it minimizes the overall entropy in its subsets. Likewise, in discretization, a good partition of the continuous variable minimizes the class entropy in the subsets of examples it creates. In [10] it was shown that optimal cut points must be between instances of distinct classes. In practical terms, for all possible partitions the class information entropy is calculated and compared with the entropy without partitions. This can be done recursively until some stopping criterion is satisfied. The stopping criteria can be defined by a user or by a heuristic method like MDLP.

\section{Discretization for Label Ranking}

A supervised discretization method for LR should take into account the properties of rankings as target variables. In this work, we propose an adaptation of the Shannon entropy for rankings. This entropy will be used in conjuction with MDLP as stopping criterion, the same way it is used for classification. First we describe our adaptation of the entropy for rankings and then we show how to integrate it with MDLP.

The entropy of classes presented in [10], which derives from the Shannon entropy, is defined as:

$$
\operatorname{Ent}(S)=-\sum_{i=1}^{k} P\left(C_{i}, S\right) \log \left(P\left(C_{i}, S\right)\right)
$$

where $P\left(C_{i}, S\right)$ stands for the proportion of examples with class $C_{i}$ in a subset $S$ and $k$ is the total number of classes in $S$.

$$
P\left(C_{i}, S\right)=\frac{\# C_{i}}{N}
$$

$N$ is the number of instances in the subset $S$.

As shown in equation 2 the Shannon entropy of a set of classes depends on the relative proportion of each class. 


\subsection{Entropy of rankings}

In this section, we explain how to adapt the entropy of classes used in [10] for LR. We start by motivating our approach with a discussion of the use in LR of the concept of entropy from classification. We then show in detail our heuristic adaptation of entropy for rankings.

To better motivate and explain our approach, we introduce a very simple synthetic dataset, $D_{e x}$, presented in Table 2 . In this test dataset we have eight distinct rankings in the target column $\pi$. Even though they are all distinct, the first five are very similar (the label ranks are mostly ascending), but very different from the last three (mostly descending ranks). Without any further considerations, it is natural to assume that an optimal split point for $D_{e x}$ should lie between values 0.5 and 0.6 (instances 5 and 6 ).

Table 2. Example dataset $D_{e x}$ : Small artificial dataset with some noise in the rankings

\begin{tabular}{l|lc|l} 
TID & Att & $\pi$ & $\lambda$ \\
\hline $\mathbf{1}$ & 0.1 & $(1,2,4,3,5)$ & $\mathrm{a}$ \\
$\mathbf{2}$ & 0.2 & $(1,2,3,4,5)$ & $\mathrm{b}$ \\
$\mathbf{3}$ & 0.3 & $(2,1,3,4,5)$ & $\mathrm{c}$ \\
$\mathbf{4}$ & 0.4 & $(1,3,2,4,5)$ & $\mathrm{d}$ \\
$\mathbf{5}$ & 0.5 & $(1,2,3,5,4)$ & $\mathrm{e}$ \\
$\mathbf{6}$ & 0.6 & $(5,4,3,1,2)$ & $\mathrm{f}$ \\
$\mathbf{7}$ & 0.7 & $(4,5,3,2,1)$ & $\mathrm{g}$ \\
$\mathbf{8}$ & 0.8 & $(5,3,4,2,1)$ & $\mathrm{h}$
\end{tabular}

In the RAC approach, the rankings are transformed into eight distinct classes as shown in column $\lambda$. As the table shows, the natural split point identified earlier is completely undetectable in column $\lambda$.

As shown in equation 2, the entropy of a set of classes depends on the relative proportion of a class. If we measure the ranking proportion the same way, we get:

$$
P\left(\pi_{i}, S\right)=1 / 8, \forall \pi_{i} \in D_{e x}
$$

We adapt this concept using the same ranking distance-based approach used to adapt the support for LRAR in APRIORI-LR [7] (equation 3). In fact, a similar line of reasoning as the one in Section 2.1 can be followed here. The uncertainty associated with a certain ranking decreases in the presence of similar - although not equal - rankings. Furthermore, this decrease is proportional to that distance.

$$
P_{\pi}\left(\pi_{i}, S\right)=\frac{\sum_{j=1}^{N} s\left(\pi_{i}, \pi_{j}\right)}{\sum_{i=1}^{K} \sum_{j=1}^{N} s\left(\pi_{i}, \pi_{j}\right)}
$$

Where $K$ is the number of distinct rankings in $S$.

As in [7], we use Kendall $\tau$ and the negative correlations are ignored (section 2.1). Note that a parallel can also be established with the frequentist view 
used in entropy. Since Kendall $\tau$ is computed from the proportion of concordant pairs of labels, this can be seen as the proportion of concordant pairwise comparisons.

However, this approach alone is not enough to give a fair measure for the entropy of rankings. The entropy of the set of classes $\left\{\lambda_{1}, \lambda_{2}\right\}$ is the same as $\left\{\lambda_{1}, \lambda_{3}\right\}$ or $\left\{\lambda_{2}, \lambda_{3}\right\}$. This happens because, $\lambda_{1}$ is as different from $\lambda_{2}$ as $\lambda_{2}$ is from $\lambda_{3}$. However, in LR, distinct rankings can range from completely different to very similar. Considering these two sets:

$$
\begin{aligned}
& \text { 1) }\{(1,2,3,4,5),(1,2,3,5,4)\} \\
& \text { 2) }\{(1,2,3,4,5),(5,4,3,2,1)\}
\end{aligned}
$$

and since the ranking proportions will be the same in 1) and 2), the entropy will be the same. Also, from a pairwise-comparison point of view, the two similar rankings in set 1) match 14 pairs from a total of 15 , while the rankings in 2) do not match any.

Considering that entropy is a measure of disorder, we believe that it makes sense to expect lower entropy for sets with similar rankings and bigger entropy for sets with completely different rankings.

For this reason we propose to add an extra parameter in the formula of entropy for rankings (equation 4) to force lower values on sets of similar rankings. This means we have to adapt Shannon entropy for a set of rankings to be more sensitive to the similarity of the rankings present in the set.

$$
\operatorname{Ent}_{L R}(S)=\sum_{i=1}^{K} P\left(\pi_{i}, S\right) \log \left(P\left(\pi_{i}, S\right)\right) \log \left(Q\left(\pi_{i}, S\right)\right)
$$

where $Q\left(\pi_{i}, S\right)$ is the average similarity of the ranking $\pi_{i}$ with the rankings in the subset $S$ defined as:

$$
Q\left(\pi_{i}, S\right)=\frac{\sum_{j=1}^{N} s\left(\pi_{i}, \pi_{j}\right)}{N}
$$

For the same reason we find noise in independent variables, it is expected to observe the same phenomenon in ranking data. As the number of labels increases, we expect to observe it with more frequency, since the number of possible combinations for $k$ labels grows to $k$ !. As an example, instances 6,7 and 8 in $D_{e x}$ can correspond to observations of the same "real" ranking, say $(5,4,3,2,1)$, but with some noise.

This measure of entropy for rankings we propose here will make the discretization method more robust to noise present in the rankings. In order to support this statement we provide an analysis of the behavior of the method with induced and controlled noise.

The results presented do not include an analysis on partial orders. Given that, Kendall $\tau$ is a measure of the proportion of the concordant pairs of labels, this entropy measure can still work with partial orders, as long as there is at least one pairwise per instance. 


\subsection{MDLP for $\mathrm{LR}$}

MDLP [10] is a well known method used to discretize continuous attributes for classification learning. The method tries to maximize the information gain and considers all the classes of the data as completely distinct classes. For this reason, we believe that the latter, as is, is not suitable for datasets which have rankings instead of classes in the target.

MDLP measures the information gain of a given split point by comparing the values of entropy. For each split point considered, the entropy of the initial interval is compared with the weighted sum of the entropy of the two resulting intervals. Given an interval $S$ :

$$
\operatorname{Gain}_{L R}(A, T ; S)=\operatorname{Ent}_{L R}(S)-\frac{\left|S_{1}\right|}{N} \operatorname{Ent}_{L R}\left(S_{1}\right)-\frac{\left|S_{2}\right|}{N} \operatorname{Ent}_{L R}\left(S_{2}\right)
$$

Where $\left|S_{1}\right|$ and $\left|S_{2}\right|$ is the number of instances in the left side $\left(S_{1}\right)$ and the number of instances in the right side $\left(S_{2}\right)$ of the cut point $T$, respectively, in the attribute $A$.

After the adaptation of entropy for sets of rankings proposed in Section 4.1, Minimum Description Length Principle for Ranking data (MDLP-R) comes in a natural way. We only need to replace the entropy for rankings in the MDLP definition presented in [10], which we transcribe below:

$M D L P C$ Criterion The partition induced by a cut point $T$ for a set $S$ of $N$ examples is accepted iff

$$
\operatorname{Gain}_{L R}(A, T ; S)>\frac{\log _{2}(N-1)}{N}+\frac{\Delta_{L R}(A, T ; S)}{N}
$$

and it is rejected otherwise.

Where $\Delta_{L R}(A, T ; S)$ is equal to:

$$
\log _{2}\left(3^{K}-2\right)-\left[K \operatorname{Ent}_{L R}(S)-K_{1} E n t_{L R}\left(S_{1}\right)-K_{2} \operatorname{Ent}_{L R}\left(S_{2}\right)\right]
$$

\section{Experimental Results}

Since what we are proposing is essentially a pre-processing method, the quality of its discretization is hard to measure in a direct way. For this reason, the experimental setup is divided in two parts. In the first part we present the results obtained from controlled artificial datasets that should give an indication whether the method is performing as expected. The second part shows results of the APRIORI-LR algorithm [7] run on datasets from the KEBI Data Repository at Philipps University of Marburg [3].

Table 3 compares the intervals discretized by the MDLP-R and MDLP in dataset $D_{e x}$. As expected, since there are eight distinct rankings, the RAC approach with MDLP for classification will see eight distinct classes and break the dataset into eight intervals. MDLP-R, however, can identify the similarities of rankings, and only breaks the dataset into two intervals.

Table 4 gives a description of the benchmark datasets from KEBI Data Repository at Philipps University of Marburg [3]. 
Table 3. Discretization results using the MDLP and MDLP-R methods

\begin{tabular}{l|c|c|ccc} 
& \multicolumn{2}{|c|}{} & \multicolumn{3}{c}{ Partitions } \\
TID & Att & $\pi$ & $\lambda$ & MDLP-R MDLP \\
\hline $\mathbf{1}$ & 0.1 & $(1,2,4,3,5)$ & a & 1 & 1 \\
$\mathbf{2}$ & 0.2 & $(1,2,3,4,5)$ & b & 1 & 2 \\
$\mathbf{3}$ & 0.3 & $(2,1,3,4,5)$ & c & 1 & 3 \\
$\mathbf{4}$ & 0.4 & $(1,3,2,4,5)$ & d & 1 & 4 \\
$\mathbf{5}$ & 0.5 & $(1,2,3,5,4)$ & e & 1 & 5 \\
$\mathbf{6}$ & 0.6 & $(5,4,3,1,2)$ & f & 2 & 6 \\
$\mathbf{7}$ & 0.7 & $(4,5,3,2,1)$ & g & 2 & 7 \\
$\mathbf{8}$ & 0.8 & $(5,3,4,2,1)$ & h & 2 & 8
\end{tabular}

Table 4. Summary of the datasets

\begin{tabular}{lcccc}
\hline Datasets & type & \# examples & \#labels & \#attributes \\
\hline autorship & $\mathrm{A}$ & 841 & 4 & 70 \\
bodyfat & $\mathrm{B}$ & 252 & 7 & 7 \\
calhousing & $\mathrm{B}$ & 20640 & 4 & 4 \\
cpu-small & $\mathrm{B}$ & 8192 & 5 & 6 \\
elevators & $\mathrm{B}$ & 16599 & 9 & 9 \\
fried & $\mathrm{B}$ & 40769 & 5 & 9 \\
glass & $\mathrm{A}$ & 214 & 6 & 9 \\
housing & $\mathrm{B}$ & 506 & 6 & 6 \\
iris & $\mathrm{A}$ & 150 & 3 & 4 \\
segment & $\mathrm{A}$ & 2310 & 7 & 18 \\
stock & $\mathrm{B}$ & 950 & 5 & 5 \\
vehicle & $\mathrm{A}$ & 846 & 4 & 18 \\
vowel & $\mathrm{A}$ & 528 & 11 & 10 \\
wine & $\mathrm{A}$ & 178 & 3 & 13 \\
wisconsin & $\mathrm{B}$ & 194 & 16 & 16 \\
\hline
\end{tabular}

\subsection{Results on artificial datasets}

Results obtained with artificial datasets can give more insight about how the discretization method performs. The synthetic datasets presented in this section are variations of a simple one which has only two initial rankings $\pi_{1}=$ $(1,2,3,4,5,6,7,8,9,10)$ and $\pi_{2}=(10,9,8,7,6,5,4,3,2,1)$. To make it as simple as possible, it has only one independent variable which varies from 1 to 100 . The first 60 instances are variations of $\pi_{1}$ and the remaining are variations of $\pi_{2}$.

In order to test the advantages of our method in comparison with the RAC approach, we intentionally introduced noise in the target rankings, by performing several swaps. Each swap is an inversion of two consecutive ranks in every ranking of the data. For each ranking the choice of the pairs to invert is random. Swaps will be done repeatedly, to obtain different levels of noise. 
We performed an experiment which varies the number of swaps from 0 to 100. The greater the number of consecutive swaps, the more chaotic the dataset will be, and hence more difficult to discretize.

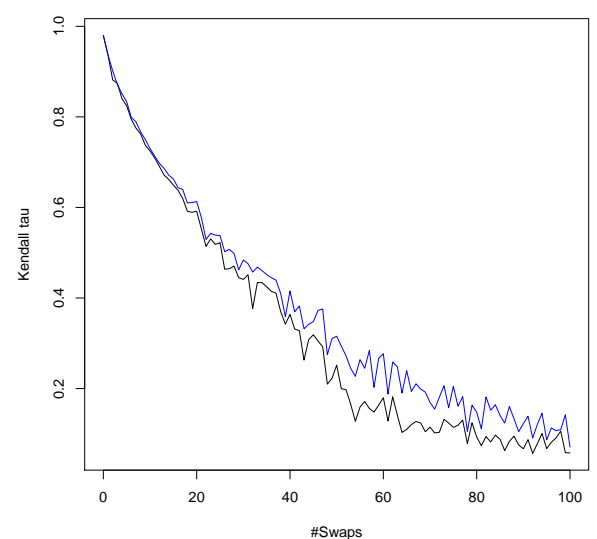

Fig. 1. Accuracy of the APRIORI-LR (expressed in terms of Kendall $\tau$ ) as a function of the number of swaps, for MDLP (black) and MDLP-LR (blue).

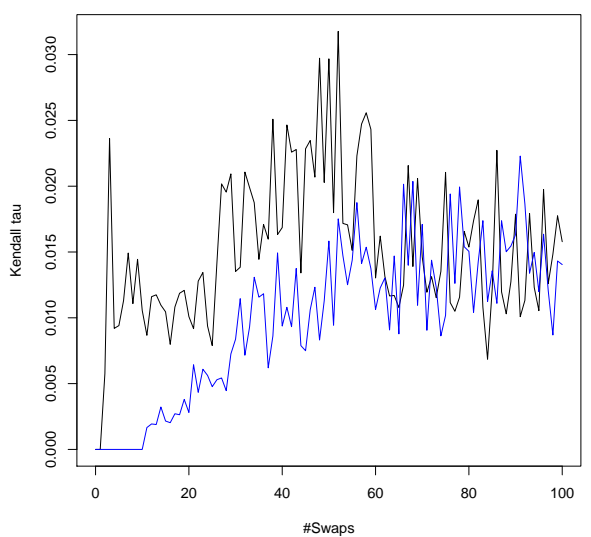

Fig. 2. Standard deviation of the accuracy of APRIORI-LR (in terms of Kendall $\tau$ ) after discretization with MDLP-R (blue) and MDLP (black).

Figure 1 compares the accuracy of APRIORI-LR with two different discretization methods, MDLP and MDLP-R. The graph, clearly indicates that the discretization with MDLP-R (blue line) leads to better results for APRIORILR, than with MDLP after a RAC transformation. While for the first cases the difference is not so evident, as the noise increases, MDLP-R gives a greater contribution.

However, if we analyze Figure 2 there is extra information in favor of MDLPR. The standard deviation of the 10 runs of the 10-fold cross-validation is zero in the presence of small amounts of noise (until approximately 10 swaps). This means that, in a scenario with reasonable noise in rankings, if one decides to use MDLP-R there are more chances to get the best result than with MDLP.

One great advantage of our method in this experiment can be seen in Figure 3. In particular, for any number of swaps until 20, our method only makes one partition which means that the split point choice is also invariant to a reasonable amount of noise. This will result in a small number of rules generated by APRIORI-LR as supported by the graph in Figure 4. In other words, MDLP-R makes APRIORI-LR much more efficient because it only needs to create approximately $1 / 10$ of the rules to obtain the same accuracy.

In Figure 5 we can see the percentage of instances from the test set that were not ranked with the default rule. Since the minimum confidence was set to $50 \%$ 


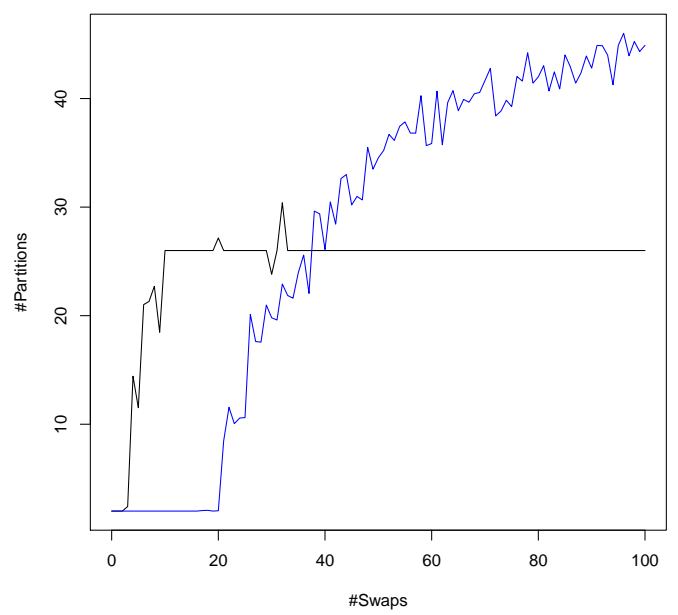

Fig. 3. Comparison of the average number of partitions generated by MDLP-R (blue) and MDLP (black)

from this graph we can conclude that ARIORI-LR with MDLP is decreasing the number of rules with confidence equal or higher than $50 \%$. Thus, MDLP-R creates more meaningful intervals which lead to higher confidence LRAR.

Additionally, in Figure 3 it is clear that, from a certain point, MDLP is no longer able to make the distinction of the target attributes and so the average number of partitions stays constant. On the other hand, the number of partitions by MDLP-R increases as the noise increases too, which is an indicator that our method is able to perform discretization even in complex situations.

\subsection{Results on benchmark datasets}

The evaluation measure is Kendall's $\tau$ and the performance of the method was estimated using ten-fold cross-validation. For the generation of Label Ranking Association Rules (LR-AR) we used CAREN [2].

The similarity parameters used for the experiments are the same as used in [7]. The minimum support (minsup) was set to $0.1 \%$. However, in some datasets, namely those with a larger number of attributes, frequent rule generation can be a very time consuming task. In this case, minsup was set to a larger value, $1 \%$ or higher.

When the algorithm cannot find at least one LRAR to rank a new instance, a default ranking is used. Since the usage of the default rule is only used as a last resourt, the minimum confidence (minconf) was adjusted with the same method used in [7] for parameter tunning. The latter aims to increase the percentage of test examples ranked without recourse to the default rule. This percentage is shown in column $M$ in Table 5.

Table 5 shows that both methods obtain similar results in benchmark datasets. As we observed in the results from artificial datasets, the number of rules 


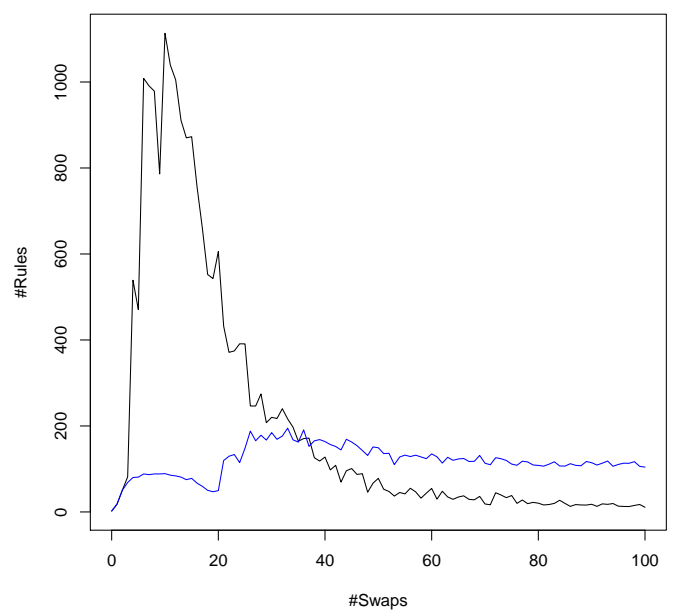

Fig. 4. Comparison of the number of rules generated by APRIORI-LR after discretization with MDLP-R (blue) and MDLP (black)

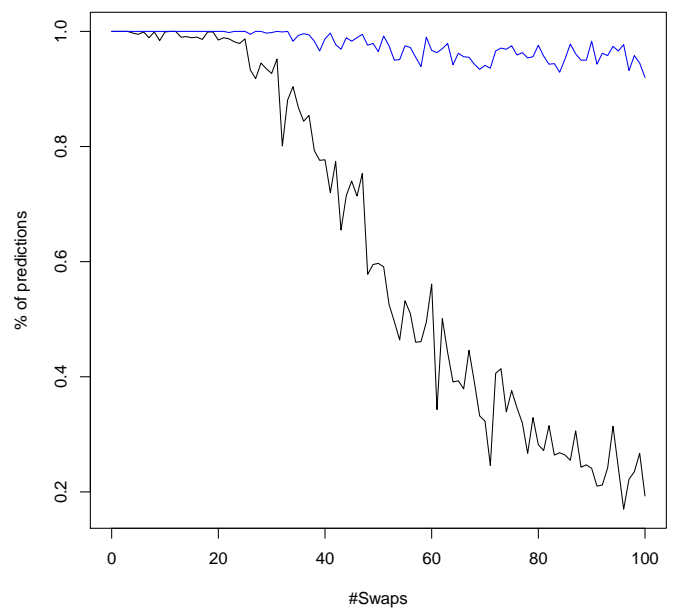

Fig. 5. Comparison of the percentage of instances from the test set that were not ranked with the default rule. MDLP-R (blue) MDLP (black)

generated after a MDLP-R discretization is higher than with MDLP in more noisy/complex datasets. This is due to a higher number of partitions. The same phenomenon is also clear in Table 5 where the number of rules is generally higher with a MDLP-R discretization.

A baseline method uses the default LRAR, which is a rule with the average ranking, and the accuracy is presented in column $\tau_{\text {baseline }}$ as show in Table 5 
Table 5. Results obtained with MDLP discretization and with $M D L P-R$ discretization on bechmark datasets

\begin{tabular}{|c|c|c|c|c|c|c|c|c|c|c|c|c|}
\hline \multirow[b]{3}{*}{ autborshin } & \multicolumn{6}{|c|}{ MDLP } & \multicolumn{6}{|c|}{ MDLP-Ranking } \\
\hline & $\tau$ & $\tau_{\text {baseline }}$ & minsup & minconf & \#rules & $\mathrm{M}$ & $\tau$ & elit & minsup & minconf & \#rules & M \\
\hline & .666 & .568 & 15 & 90 & 14975 & $100 \%$ & .691 & -568 & 1.5 & 100 & 6010 & $100 \%$ \\
\hline bodyfat & .063 & -.063 & 0.1 & 70 & 17135 & $100 \%$ & .066 & -.063 & 0.1 & 65 & 23415 & $100 \%$ \\
\hline calhousing & .329 & .048 & 0.1 & 35 & 488 & $100 \%$ & .304 & .048 & 0.1 & 30 & 1315 & $100 \%$ \\
\hline cpu-small & .418 & .234 & 0.1 & 35 & 326 & $100 \%$ & .458 & .234 & 0.1 & 40 & 3888 & $100 \%$ \\
\hline elevators & .648 & .288 & 0.1 & 60 & 291 & $98 \%$ & .670 & .288 & 0.1 & 60 & 5681 & $98 \%$ \\
\hline fried & .802 & -.002 & 0.1 & 55 & 8257 & $97 \%$ & .735 & -.002 & 0.1 & 100 & 10445 & $100 \%$ \\
\hline glass & .817 & .684 & 0.1 & 80 & 168 & $100 \%$ & .860 & .684 & 0.1 & 100 & 1686 & $100 \%$ \\
\hline ing & .779 & .053 & 0.1 & 65 & 420 & $100 \%$ & .809 & .072 & 0.1 & 70 & 1284 & $100 \%$ \\
\hline & .962 & .089 & 0.1 & 85 & 36 & $100 \%$ & .934 & .089 & 0.1 & 80 & 41 & $100 \%$ \\
\hline segment & .898 & .371 & 1 & 85 & 5110 & $100 \%$ & .890 & .371 & 1 & 80 & 2888 & $100 \%$ \\
\hline sto & .894 & .072 & 0.1 & 80 & 1197 & $100 \%$ & .890 & .072 & 0.1 & 80 & 2980 & $100 \%$ \\
\hline vehi & .847 & .179 & 0.1 & 90 & 35085 & $100 \%$ & .835 & .179 & 0.1 & 100 & 140051 & $100 \%$ \\
\hline vowe & .802 & .195 & 0.1 & 100 & 7593 & $100 \%$ & .632 & .195 & 0.1 & 100 & 15606 & $100 \%$ \\
\hline wine & .937 & .329 & 0.1 & 100 & 1192 & $100 \%$ & .877 & .329 & 0.1 & 100 & 3666 & $100 \%$ \\
\hline wisconsin & .321 & -.031 & 0.1 & 100 & 18223 & $100 \%$ & .271 & -.031 & 0.1 & 100 & 16799 & $100 \%$ \\
\hline
\end{tabular}

\section{Conclusions}

In his paper we present a simple adaptation of the supervised discretization method, MDLP, for LR. This work was motivated by the lack of supervised discretization methods to deal with rankings in the target variable. The results clearly show that this is a viable LR method.

Our method clearly outperforms MDLP in the experiments with artificial data. In this work we empirically show that, in simple scenarios, MDLP-R deals with noisy ranking data accordingly to expected. Hence the latter is more reliable, in this kind of situation, than MDLP.

In Section 5.1 there are two sides of the new MDLP-R. In the presence of very simple LR problems (\#swaps $\leq 20$ ), it has less partitions and APRIORILR generates fewer rules than with MDLP. On the other hand, in more complex situations (\#swaps > 20) it has more partitions than MDLP and, consequently, APRIORI-LR creates more rules. The latter, in our opinion, cannot be seen as a disadvantage. The fact that there are more partitions being made means that the method is still able to identify similar groups of rankings even in very complex cases.

We believe that the measure of entropy for rankings proposed here, despite its heuristic nature, makes sense and its useful in the LR field. This new measure and MDLP-R bring new possibilities for processing ranking data and can motivate the creation of new methods for LR learning that cannot deal with continuous data. Furthermore, even though it was developed in the context of the LR task, it can be also applied to other fields such as regression since it is based on a distance measure such as Kendall $\tau$.

This work uncovered several possibilities that could be better studied in order to improve the discretization in the LR field. They include: the choice of parameters in the stopping criterion; the usage of other entropy measures for rankings.

We also believe that it is essential to test the methods on real LR problems like metalearnig or predicting the rankings of financial analysts [1]. The KEBI datasets are adapted from UCI classification problems. In terms of real 
world applications, these can be adapted to rank analysts, based on their past performance and also preferences of radios, based on user's preferences.

\section{Acknowledgments}

This work was partially supported by Project Best-Case, which is co-financed by the North Portugal Regional Operational Programme (ON.2 - O Novo Norte), under the National Strategic Reference Framework (NSRF), through the European Regional Development Fund (ERDF).

We thank the anonymous referees for useful comments.

\section{References}

1. A. Aiguzhinov, C. Soares, and A. P. Serra. A similarity-based adaptation of naive bayes for label ranking: Application to the metalearning problem of algorithm recommendation. In Discovery Science, pages 16-26, 2010.

2. P. J. Azevedo and A. M. Jorge. Ensembles of jittered association rule classifiers. Data Min. Knowl. Discov., 21(1):91-129, 2010.

3. W. Cheng, J. Hühn, and E. Hüllermeier. Decision tree and instance-based learning for label ranking. In ICML '09: Proceedings of the 26th Annual International Conference on Machine Learning, pages 161-168, New York, NY, USA, 2009. ACM.

4. W. Cheng and E. Hüllermeier. Label ranking with abstention: Predicting partial orders by thresholding probability distributions (extended abstract). CoRR, abs/1112.0508, 2011.

5. W. Cheng, E. Hüllermeier, W. Waegeman, and V. Welker. Label ranking with partial abstention based on thresholded probabilistic models. In Advances in Neural Information Processing Systems 25, pages 2510-2518, 2012.

6. D. K. Y. Chiu, B. Cheung, and A. K. C. Wong. Information synthesis based on hierarchical maximum entropy discretization. J. Exp. Theor. Artif. Intell., 2(2):117-129, 1990.

7. C. R. de Sá, C. Soares, A. M. Jorge, P. J. Azevedo, and J. P. da Costa. Mining association rules for label ranking. In PAKDD (2), pages 432-443, 2011.

8. J. Dougherty, R. Kohavi, and M. Sahami. Supervised and unsupervised discretization of continuous features. In Machine Learning - International Workshop Then Conference -, pages 194-202, 1995.

9. T. Elomaa and J. Rousu. Efficient multisplitting revisited: Optima-preserving elimination of partition candidates. Data Min. Knowl. Discov., 8(2):97-126, 2004.

10. U. M. Fayyad and K. B. Irani. Multi-interval discretization of continuous-valued attributes for classification learning. In IJCAI, pages 1022-1029, 1993.

11. M. Gurrieri, X. Siebert, P. Fortemps, S. Greco, and R. Slowinski. Label ranking: A new rule-based label ranking method. In IPMU (1), pages 613-623, 2012.

12. M. Kendall and J. Gibbons. Rank correlation methods. Griffin London, 1970.

13. S. Kotsiantis and D. Kanellopoulos. Discretization techniques: A recent survey. GESTS International Transactions on Computer Science and Engineering, $32(1): 47-58,2006$.

14. B. Liu, W. Hsu, and Y. Ma. Integrating classification and association rule mining. Knowledge Discovery and Data Mining, pages 80-86, 1998. 
15. G. Ribeiro, W. Duivesteijn, C. Soares, and A. J. Knobbe. Multilayer perceptron for label ranking. In ICANN (2), pages 25-32, 2012.

16. C. Spearman. The proof and measurement of association between two things. American Journal of Psychology, 15:72-101, 1904. 